\title{
Using the Integrated Vehicle Health Management Research Test and Integration Plan Wiki to Identify Synergistic Test Opportunities
}

\author{
Syri J. Koelfgen* \\ NASA Dryden Flight Research Center, Edwards, CA 93523 \\ James J. Faber ${ }^{\dagger}$ \\ TYBRIN Corporation, NASA Dryden Flight Research Center, Edwards, CA 93523
}

\begin{abstract}
The National Aeronautics and Space Administration (NASA) and the aviation industry have recognized a need for developing a method to identify and combine resources to carry out research and testing more efficiently. The Integrated Vehicle Health Management (IVHM) Research Test and Integration Plan (RTIP) Wiki is a tool that is used to visualize, plan, and accomplish collaborative research and testing. Synergistic test opportunities are developed using the RTIP Wiki, and include potential common resource testing that combines assets and personnel from NASA, industry, academia, and other government agencies. A research scenario is linked to the appropriate IVHM milestones and resources detailed in the wiki, reviewed by the research team members, and integrated into a collaborative test strategy. The scenario is then implemented by creating a test plan when appropriate and the research is performed. The benefits of performing collaborative research and testing are achieving higher Technology Readiness Level (TRL) test opportunities with little or no additional cost, improved quality of research, and increased communication among researchers. In addition to a description of the method of creating these joint research scenarios, examples of the successful development and implementation of cooperative research using the IVHM RTIP Wiki are given.
\end{abstract}

\section{Nomenclature}

$\begin{array}{ll}\text { API } & =\text { Associate Principal Investigator } \\ \text { COTS } & \text { commercial off the shelf } \\ \text { DFRC } & =\text { Dryden Flight Research Center } \\ \text { EMA } & =\text { electromechanical actuator } \\ \text { FLEA } & =\text { Flyable Electromechanical Actuator } \\ \text { IVHM } & =\text { Integrated Vehicle Health Management } \\ \text { LaRC } & =\text { Langley Research Center } \\ \text { MOSFET } & =\text { metal oxide semiconductor field-effect transistor } \\ \text { NASA } & =\text { National Aeronautics and Space Administration } \\ \text { PET } & =\text { Prognostics Electronic Test } \\ \text { PHM } & =\text { Prognostic Health Management } \\ \text { RTI } & =\text { Research Test and Integration } \\ \text { RTIP } & =\text { Research Test and Integration Plan } \\ \text { RUL } & =\text { remaining useful life } \\ \text { TAMS } & =\text { Tributary Analysis Monitoring System } \\ \text { TRL } & =\text { Technology Readiness Level }\end{array}$

\footnotetext{
* Aerospace Engineer, Aerodynamics \& Propulsion Branch, P.O. Box 273/MS 2228, Edwards, CA, AIAA Senior Member.

${ }^{\dagger}$ Aerospace Engineer, Aerodynamics \& Propulsion Branch, NASA DFRC, P.O. Box 273/MS 4840B, Edwards, CA, AIAA Senior Member.
} 


\section{Introduction}

There is a need within the National Aeronautics and Space Administration (NASA) and the aviation industry to identify and combine resources and personnel to perform research and testing more efficiently. ${ }^{1,2}$ A collaborative research approach leverages the expertise of multiple team members, reduces the number of required man hours to accomplish the work, reduces the budget, and allows personnel to more efficiently achieve research goals by using shared resources such as laboratories and test aircraft. This pooling of resources and personnel for research and test activities has the possibility of creating synergistic effects that can include:

1) increasing the Technology Readiness Level (TRL) for test activities with little or no additional cost to perform the tests,

2) improving the quality of research activities by increasing the quantity of data sets available to researchers through the acquisition of test data via ride-along test activities which add little or no additional cost, and

3) increasing the overall knowledge base by improving communication among principal investigators and research partners while reducing the duplication of work.

In order to perform this type of collaborative research work, a process for developing cooperative research is needed. This paper introduces the method for identifying and developing joint test strategies using the Integrated Vehicle Health Management (IVHM) Research Test and Integration Plan (RTIP) Wiki. The IVHM RTIP Wiki is an interactive web-based platform for providing information on the integration, test, and reporting of research being performed in support of the NASA IVHM Project. In this paper, the RTIP Wiki is described at high level, the process for developing joint research scenarios is explained, and examples of the successful development and implementation of research tests using the IVHM RTIP Wiki are presented.

\section{Research Test and Integration Plan}

The RTIP is the compilation of the milestones, requirements, resources, test strategies, and results of the technologies being researched by the NASA Aviation Safety Program's IVHM Project. The objective of the IVHM Project is to develop validated tools, technologies, and techniques for the automated detection, diagnosis, prognosis, and mitigation of adverse events that can occur during flight. ${ }^{3}$ Adverse events include system, subsystem, or component faults or failures that can develop from in-flight damage, degradation, or environmental hazards. The real-time mitigation of adverse events leads to safer flight operations and an increase in the remaining useful life (RUL) of the flight system and vehicle. The IVHM Project is divided into five subject matter themes, consisting of detection, diagnosis, prognosis, mitigation, and integrity assurance; all of which are focused on recognizing and dealing with previous adverse events that have the potential to reoccur. Each of these five themes is assigned a cognizant engineer who coordinates the RTIP documentation and test activities with the Associate Principal Investigator (API) overseeing each theme. IVHM research milestones are outlined in the IVHM Technical Plan, ${ }^{3}$ which receives input from the NASA Aviation Safety Program, the Decadal Survey of Civil Aeronautics, ${ }^{4}$ the National Plan for Aeronautics Research and Development and Related Infrastructure, ${ }^{5}$ the Next Generation Air Transportation System Research and Development Plan, ${ }^{6}$ and other contributions from NASA, industry, academia, and other government agencies. The milestones from the technical plan are then organized into the RTIP in order to facilitate a means of meeting the IVHM goals.

The RTIP has been assembled into a web-based portal called a wiki, which is an online, interactive document. This was done to help researchers learn what is available across NASA Centers and meet the milestones established in the IVHM Technical Plan. A screenshot of the RTIP Wiki is shown in Fig. 1. The RTIP provides the framework for the planning and coordination needed to understand, demonstrate, and communicate the overall state and direction of the IVHM Project. It formally tracks cross-project test activities and provides for the traceability of requirements. The RTIP leverages the IVHM team's diverse competencies in test planning, systems engineering, and analysis. The RTIP Wiki contains seven sections: (1) Introduction, (2) Test and Integration Overview, (3) Test and Integration Theme Level, (4) Capabilities and Assets for IVHM Research Test and Integration (RTI), (5) Integration, (6) Results Reporting, and (7) Attachments. 


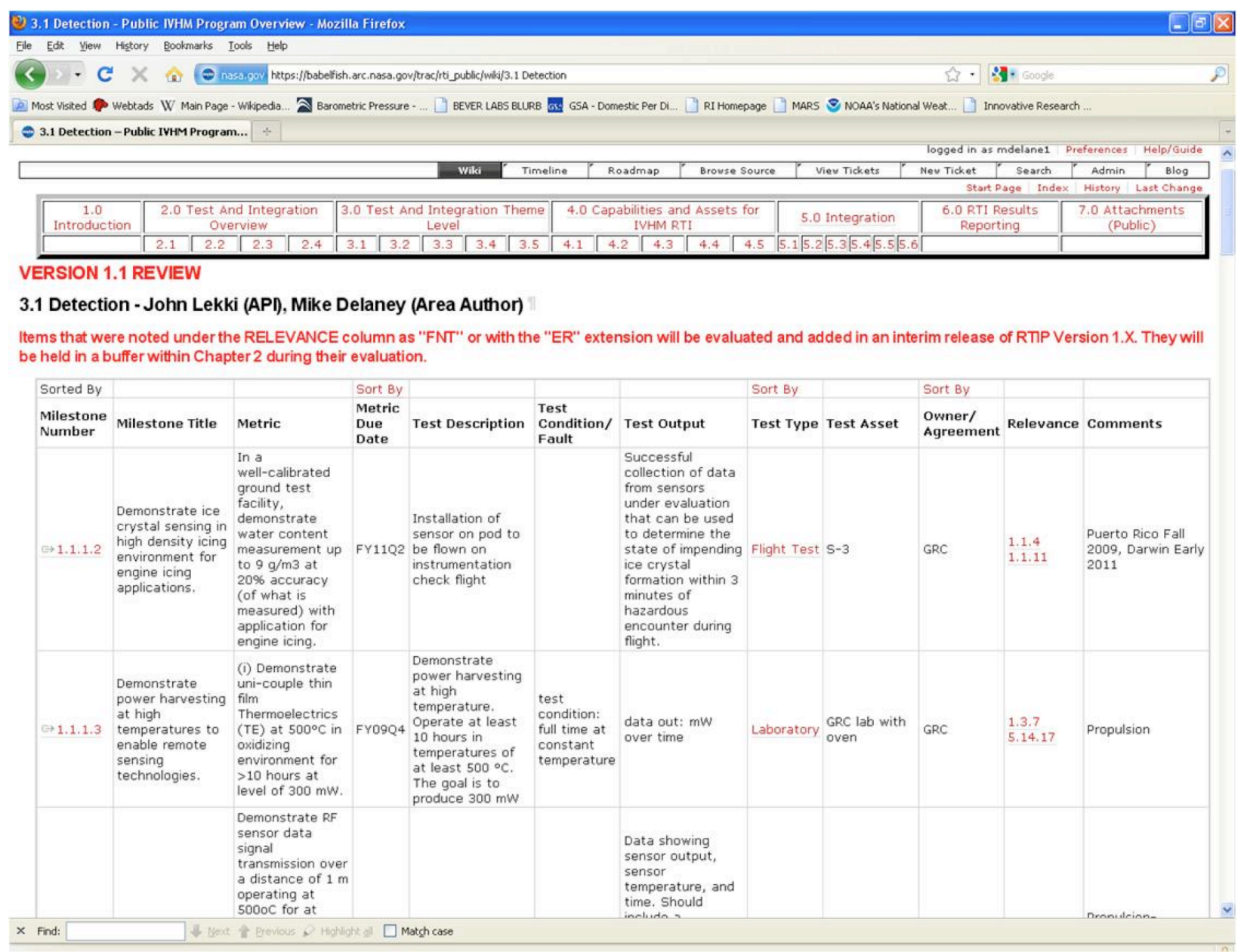

Figure 1. RTIP Wiki screenshot.

The Introduction section of the RTIP Wiki describes the responsibilities of team members in the development and maintenance of the RTIP Wiki, and how the duties of the team are assigned for each IVHM theme described in Section 3 of the RTIP. This section also defines the scope of the RTIP Wiki and outlines the organization of the seven wiki sections.

Section 2 of the RTIP Wiki is the Test and Integration Overview. It contains four subsections: (2.1) System Architecture; (2.2) Adverse Events, Technology Needs and IVHM Portfolio; (2.3) Enabling IVHM Technologies for Future Aircraft; and (2.4) Integrated Architecture Assessment Working Group. The System Architecture subsection describes the process for the creation of the IVHM Project goals and the team responsible for this effort. The Adverse Events, Technology Needs and IVHM Portfolio subsection contains information on broad category adverse events that have been factors in past aircraft accidents. The adverse events are mapped to a portfolio of technology requirements that IVHM research is attempting to address, as given by the project milestones. Additionally, the adverse events are mapped to the technology needs that currently planned IVHM research does not address. The Enabling IVHM Technologies for Future Aircraft subsection lays out in table format how future aviation technology concepts could be realized through the research and development of IVHM propulsion, aircraft, airframe, and software engineering tools. Finally, the Integrated Architecture Assessment Working Group subsection details the intent and charter of the group, and identifies the working group members.

The third section of the RTIP Wiki is the Test and Integration Theme Level, which contains five subsections: (3.1) Detection, (3.2) Diagnosis, (3.3) Prognosis, (3.4) Mitigation, and (3.5) Integrity Assurance. Each of the subsections is a category for classifying how the various IVHM research activities are utilized to manage vehicle health. The Detection subsection contains a table that details various research milestones within the theme. The objective of the detection theme is to develop validated technologies that will allow for the detection of anomalies 
from adverse events. The Diagnosis subsection contains a table that describes milestones addressing the theme. The goal of the diagnosis theme is to develop integrated and validated technologies that will determine the causal factors of an adverse event, the nature and severity of the event, and the distinguishing features of the event within a family of potential adverse events. This diagnostic capability goes beyond standard fault isolation techniques and relies on the detection theme to provide evidence of the anomaly. The Prognosis subsection contains a table describing a variety of research milestones within its theme. The aim of the prognosis theme is to determine a validated estimate of the RUL of the systems affected by candidate failures. Information gathered from both detection and diagnosis health management systems aids in the prognosis of the RUL. The Mitigation subsection contains a table outlining research milestones focusing on its theme. The goal of the mitigation theme is to develop onboard mitigation technologies that minimize the impact of adverse effects, as identified by deployed detection, diagnosis, and prognosis systems, to ensure continued safe flight and/or landing of the aircraft. The Integrity Assurance subsection contains a table describing various research milestones that address the theme. The objective of the integrity assurance element is to develop advanced integrity assurance tools, test beds, and technologies for assessing the performance, robustness, and other integrity assurance needs required for the safe deployment of IVHM systems.

Section 4 of the RTIP Wiki describes the capabilities and assets for IVHM RTI, and contains five subsections: (4.1) Laboratory, (4.2) Test Fixture, (4.3) Ground Test, (4.4) Flight Test, and (4.5) Other. Each of these subsections is a category for classifying how the various IVHM research activities are tested, and in many cases reflects the TRL of the research. The Laboratory subsection contains a table that describes laboratory facilities that may be utilized for various IVHM research activities. For the purposes of IVHM research, basic technological elements are integrated in the laboratory environment to establish that the pieces will work together. Data is also recorded to determine whether components are working. The Test Fixture subsection contains a table outlining test fixture facilities available for IVHM research. In the IVHM test fixture environment, components and supporting elements are integrated such that a technology can be tested in a simulated environment. The Ground Test subsection has a table detailing various ground test facilities or vehicles that can be used for IVHM research. A representative model or prototype system is tested in the ground test environment, which is well beyond the breadboard laboratory-type test. The ground test represents a major step up in the demonstrated readiness of a technology. The table in the Flight Test subsection describes flight-test vehicles available for IVHM research. In the flight-test environment, a system prototype is demonstrated in an operational flight environment, such as in an aircraft. The Other subsection contains a table that outlines various testing facilities or technologies that are utilized for IVHM research activities, but are not categorized as a laboratory, test fixture, ground test facility or flight-test facility. In addition to describing the test facilities, one of the purposes of Section 4 in the RTIP Wiki is to share knowledge of the testing assets available across NASA Centers with the intention of making the assets available for use by researchers previously unaware of their existence. The test facilities that fall under this classification tend to be highly customized test set ups and may encompass technologies that could be useful for other research activities, but do not require the sharing of hardware or facilities. Examples of such test assets include data-mining techniques and battery prognostic routines.

Section 5, the Integration section of the RTIP Wiki, documents the efforts the RTIP team has made in creating common resource research and testing. Early in their development, common resource testing ideas are called research scenarios. These research scenarios move forward if they are seen to have worthwhile merit among the interested research partners. Other research scenarios will not move beyond the concept stage for a multitude of reasons including: funding, academic interest, difficulty of implementation, etc. In any case, the research scenarios are documented in Section 5.

The last two sections of the wiki are for IVHM research documentation. The sixth section, Results Reporting, serves as a depository of results, reports, and papers from IVHM research testing. Section 7 of the RTIP Wiki houses attachments, and contains publicly released documents pertaining to the RTIP Wiki, as well as private attachments which have controlled access.

The RTIP identifies IVHM milestones, documents available assets and resources, provides milestone due dates, and proposes testing concept needs. Thus the RTIP is a collaborative tool that provides a communication venue for each of the APIs to describe and convey their respective requirements to integrate, test, and validate IVHM research topics. An integral part of the RTIP is the documentation of established laboratories, aircraft, and other test facilities within NASA, other government agencies, industry, and university partners that would be useful for researching IVHM milestones. To allow for the incorporation of new test facilities that may become available throughout the course of the project, the RTIP has the flexibility of being a living document that may be updated as the need arises. The RTIP is formally provided to the IVHM Project in the second and fourth quarter of each year. New test facilities may be incorporated into the RTIP for a variety of purposes, including cost effectiveness, additional capabilities, or joint cross-project or cross-program activities. The output of the wiki includes identified integration and testing 
opportunities; established partnerships with other government agencies, industry, and academia; support for resources and assets; and potential applications to other projects.

\section{Identifying Synergistic Test Opportunities Using the RTIP}

\section{A. Background and Motivation for Developing Collaborative Test Plans}

Prior to developing any combined resource test activities, the RTI team visited the NASA Centers involved in IVHM work to meet with the researchers and APIs. During the visits, meetings were held for the RTI team to update the status of the various IVHM research activities and their associated milestones, and to tour the facilities and laboratories at the individual Centers to collect contact information and basic capabilities. During these meetings, the APIs and researchers identified milestones called seed milestones that required additional resources and facilities to be accomplished. With the list of seed milestones in hand, the RTI team started to look for available test assets that would enable these milestones to be accomplished. While developing the test strategies for the seed milestones, the RTI team also found additional IVHM research activities and milestones that could be aided by utilizing the same test activity and asset identified for the seed milestones. This led to the need for generating a collaborative research and test approach for the seed milestones. The IVHM RTIP Wiki was created as a means to foster and meet this need for performing joint research.

As previously noted, IVHM research falls under one of the five subject matter themes. The RTIP Wiki takes an IVHM Technical Plan milestone within a theme and links it to the requirements and adverse events for the milestone that have been established by the IVHM Project. The capabilities and assets that may be used to carry out IVHM research are also listed and described in the RTIP Wiki. In order to identify and develop a collaborative test strategy for researching the topic detailed in each milestone, a process for creating cooperative test plans is necessary. Team members, resources, and facilities necessary for investigating certain research topics may not be in the same location, which increases the complexity of developing such a joint test plan. The method developed and currently being put into practice by the IVHM Project is to use the RTIP Wiki to identify synergistic test opportunities. The benefit of performing this type of joint research and testing is that the sum of the pooled assets, resources, personnel, and budgets is greater than the individual contributions. The payoff may be the realization of a higher TRL research opportunity than that which a single entity could produce in the same amount of time, with little or no additional cost. Examples of the time and cost savings benefits are seen in a ride-along collaborative flight test, where an IVHM experiment is allowed to fly on an aircraft that is already being flown for another purpose, such as a pilot proficiency flight. The pilot's organization is already funding the flight for the pilot's training, and the experiment is allowed to ride on the aircraft at little or no cost to the IVHM Project. This joint flight also saves time in that the experiment does not require its own dedicated research flight and can be scheduled to fly during a routine proficiency flight. Improved communication among researchers is another benefit of combined testing, which leads to the sharing of knowledge and a reduced duplication of research efforts, which increases the overall efficiency of performing research.

\section{B. Generating Synergistic Test Scenarios}

The IVHM RTIP test team is lead by NASA Dryden Flight Research Center (DFRC) personnel. DFRC assigns a team member, otherwise referred to as the cognizant engineer, to each of the five IVHM themes. The cognizant engineers coordinate documentation and test activities with the API, who is typically from a different NASA Center, assigned to the same theme. This collaborative effort provides a communication venue and the synergy needed for each of the APIs to document and convey their respective requirements and high-level methods to validate, test, and integrate research topics. As a result of the coordination and communication essential in performing joint research, the cognizant engineer gains a high-level understanding of the wants, needs, and capabilities of each of the researchers, APIs, and NASA Centers with which they work. The high level of familiarity and the process of keeping the RTIP Wiki updated aid the cognizant engineer in developing potential research scenarios.

The RTIP Wiki is used to create collaborative test plans. Test strategies are integrated into the wiki by first developing a research scenario that is made available to and reviewed by team members who will be involved in conducting the research. The scenario includes potential common resource tests that combine assets and personnel from NASA, industry, academia, and other government agencies. For example, common resource testing may be in the form of utilizing a single flight test to perform dual functions, such as a proficiency flight for a pilot where a researcher rides along to gather data on a flight system of interest. After a common resource test scenario is developed, it is posted on the RTIP Wiki in Section 5 and linked to the appropriate IVHM milestones and resources detailed in the wiki. After issues related to conducting the research are reviewed and resolved by the research team members, the proposed research scenario is integrated into a collaborative test strategy. The test plan is then 
implemented and the research is performed. As previously described, the wiki is also used for communicating results and tracking the progress of the research.

\section{Performing Collaborative Research and Testing}

The process of utilizing the RTIP Wiki to generate and conduct combined test efforts has been successfully put into practice. The IVHM RTIP Wiki has been used to develop several research scenarios, integrate team members and resources, and build test plans. One such successful example is the integration of an aging electronics and actuator health management research scenario into a flight test. The aging electronics/actuator research will be conducted in Spring 2010 on a flight test in ride-along fashion for little added cost to the IVHM Program. The TRL of the research will be increased by placing the test equipment in an actual flight environment, which will subject the experimental fixtures to in-flight temperatures, pressures, vibrations, etc. The test equipment will also be allowed to access actual actuator position data from the aircraft during flight. In addition to this test, other research scenarios have been developed using the IVHM RTIP Wiki.

Figure 2 shows both currently approved and proposed collaborative test scenarios that have been put together using the RTIP Wiki. The tests in the figure include an engine ground test, a fleet level data-mining effort, an electromechanical actuator (EMA) flight test, a structural health monitoring flight test, and an aging electronics/actuator health management test. Each test scenario and the use of the RTIP Wiki in developing it are described in turn.

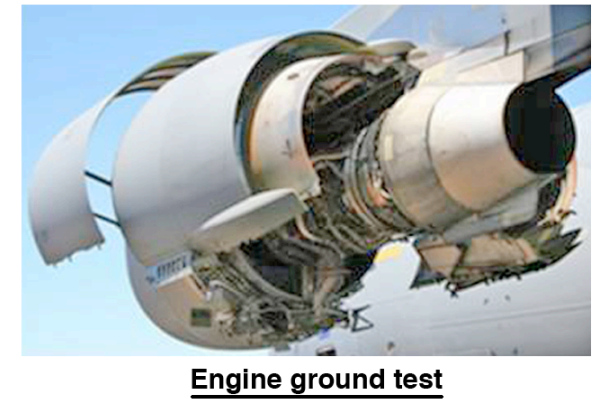

- Utilizing commercial derivative turbofan

- Addressing detection, diagnosis, and prognosis

- Researching mechanical and gas path sensor enhancements

- Collaboration with multiple NASA centers and industry partners

\section{EMA flight test}

- Addressing prognosis

- Installing EMA subjected to air loads

- Seeding faults within EMA and predicting RUL to failure with research algorithms

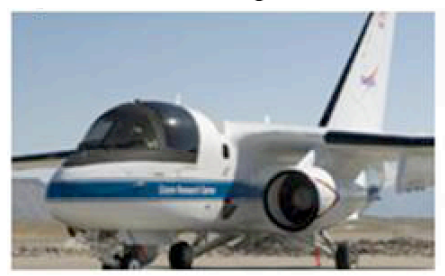

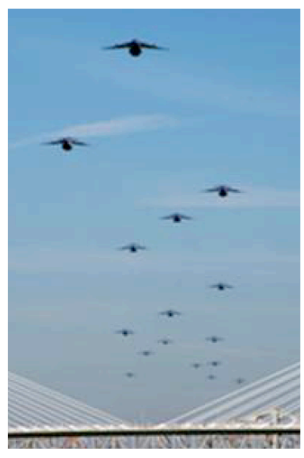

Fleet level data mining

- Utilizing transport category aircraft

- Collecting of large data sets from multiple aircraft in Tbyte range

- Collaboration with multiple NASA centers and industry partners

Figure 2. Research scenarios developed using the IVHM RTIP Wiki.

\section{A. Engine Ground Test}

A collaborative engine ground test scheduled for late 2010 or early 2011 is currently using the RTIP Wiki for its planning, integration, and implementation. The research scenario was first developed using the wiki by mapping two IVHM milestones to a test asset that could be used to carry out the research. In this case, the milestones were to demonstrate multiple sensor technologies that will enable the detection of faults in propulsion structural health monitoring systems and to demonstrate the operation of multiple high-temperature sensors that will enable improved 
gas path performance diagnostics. ${ }^{3}$ The asset was a commercial derivative turbofan engine, shown in the upper left corner of Fig. 2. Using the particular engine desired for the ground test required the collaboration among multiple NASA Centers, industry partners, and other government organizations; as the engine is located on an aircraft owned by a non-NASA entity and was developed by yet another entity in the commercial aviation industry.

The test concept and test plan were generated and populated in the RTIP Wiki, and reviewed by the research team members. The test itself is a sensor and algorithm aircraft ground test focused on meeting a propulsion health management IVHM milestone. The research will investigate the use of a fan and turbine microwave blade tip clearance sensor, a self-diagnosing vibration sensor, and an emission sensor to detect faults in an actual engine ground test, whereas previously these sensors have only been tested in the laboratory. The engine will be operated normally with seeded fault inputs (incipient and fast progression) in both the mechanical and gas path environments. Nominal operation during the ground test will include engine runs with power sweeps. Potential faults to be entered will include an induced fan imbalance (the mechanical environment), stability bleed fail open (the engine gas path), and a turbine case clearance control (the gas path). Engine models will be embedded in various computer platforms and run real-time in a data rack (Fig. 3) mounted inside the aircraft during the ground test. This will allow the user to view, trim, and adjust the model during the test. The data will also be stored for post-run data reduction and analysis. The RTIP Wiki has been found to be a useful tool in planning the research. It allows the team to communicate the goals of the research, steam line the steps necessary for completing the work, and identify the resources required for the test.

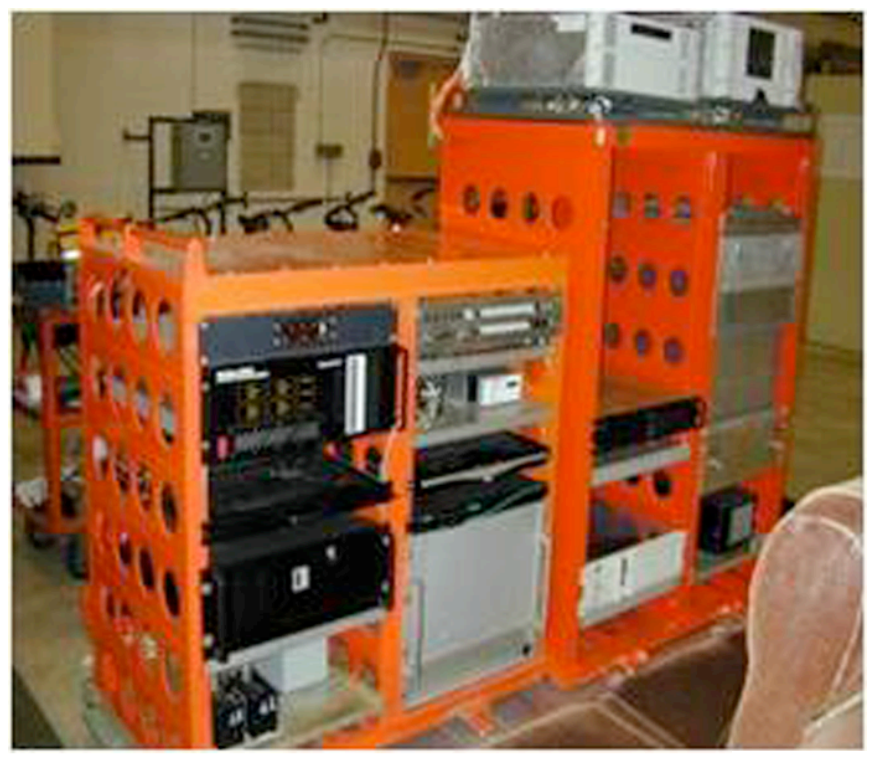

Figure 3. Data rack.

\section{B. Fleet Level Data Mining}

Another current research effort utilizing the RTIP Wiki for implementation is an aircraft fleet-level data-mining endeavor. The effort involves testing algorithms for the detection, diagnosis, and prediction of faults with operational data from a fleet of aircraft. The current research focuses on fault prediction algorithms tested on data from commercial aircraft fleets, while future endeavors will include military fleets with both new and aged aircraft data. Since data mining research requires access to data sets from operational aircraft fleets involving a large number of aircraft, many players will be engaged in the implementation of the research. The players include NASA, the airline industry, commercial aviation producers, other government agencies, etc. The RTIP Wiki tools will be used to integrate the joint research effort by identifying adverse events, linking theme and milestone relevance to the work, finding the assets and resources required to complete the effort, planning the implementation of the research, and housing the documentation. The wiki's online format will allow the research team members, many of whom are not located in the same area, to generate a synergistic plan for conducting the work. 


\section{EMA Flight Test}

An EMA flight test addressing the IVHM theme of prognosis is also using the RTIP Wiki for integration. This research addresses the IVHM milestones to validate prognostic and RUL estimation models for EMAs, to validate methodologies and tools for the diagnosis and prognosis of failures associated with aircraft components and subsystems implicated by adverse events, and to demonstrate improved IVHM using models with varying operating conditions. ${ }^{3}$ The research will make use of the Flyable Electromechanical Actuator (FLEA) test stand (Fig. 4), which will provide an opportunity to validate models and algorithms for damage propagation and the estimation of the RUL of aircraft systems. The FLEA test stand contains two test actuators (one fault injected and one nominal, which may be switched in flight), as well as one load actuator scaled to the air load. The top shelf of the test stand contains the actuators; and the bottom shelf houses a commercial off the shelf (COTS) laptop computer, a power supply, and a motor control board. The equipment will be tested in the presence of vibrations, noise, gravity-loads, and temperature changes during flight. Nominal and off-nominal data will be collected under the same flight conditions. The sensor data will be routed to the Prognostic Health Management (PHM) system for fault diagnosis and RUL prediction. The FLEA test stand will allow the testing of EMA fault detection and prognosis technologies in a flight environment and will substantially increase their TRLs, all without the expense of dedicated flights due to the collaborative efforts; the stand is designed to function as a non-intrusive secondary payload. Additionally, the test allows actuators to be flight-tested before placing them in actual airflow. The research will demonstrate real-time capabilities of the data acquisition system and health management models. The RTIP Wiki is being used to link the IVHM milestones to the resources and test assets needed to perform the work, to integrate and implement the test plan, and to report the results of the EMA test. For this particular research, the wiki has been especially useful in documenting discussions among multiple NASA Centers on the various flight platforms that are available to perform the research.

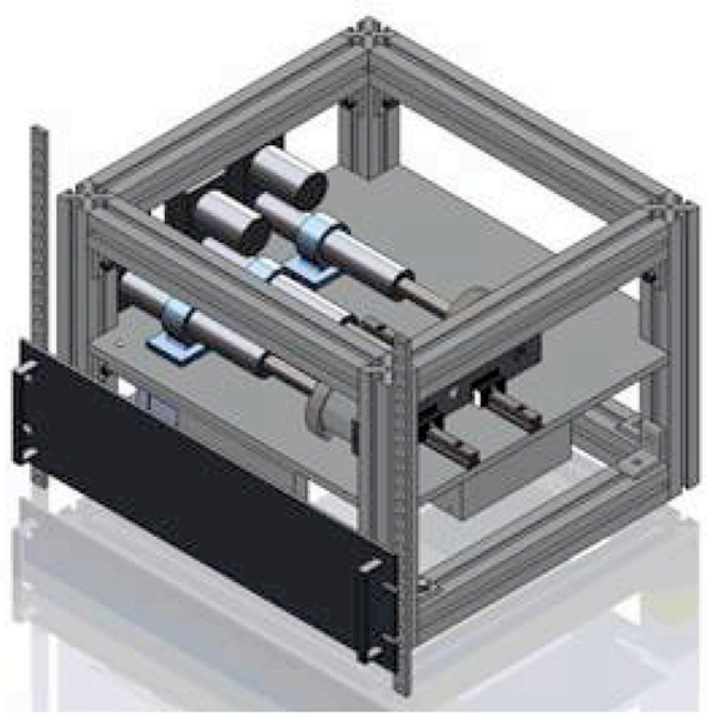

Figure 4. FLEA test stand.

\section{Aging Electronics/Actuator Health Management}

Another approved research effort developed using the RTIP Wiki addresses the IVHM diagnosis and prognosis themes. The Prognostics Electronic Test (PET) is being developed to provide an opportunity to validate models and algorithms for predicting damage propagation and estimating the RUL of aircraft systems. The PET consists of a test flight aimed at assessing EMA fault detection and prognosis technologies in a flight environment using a Ridgetop Group Incorporated (Tucson, Arizona) EMA emulator (Fig. 5). The PET includes a suitcase EMA emulator that will function as a non-intrusive secondary payload during flight. The EMA emulator is comprised of two motors and two actuators (one load actuator and one test actuator). Both new and aged metal oxide semiconductor field-effect transistors (MOSFETs) will be tested during flight, while nominal and off-nominal data will be collected under the same flight conditions. Motion and load profiles will be derived in real-time from one of the aircraft actuators during the flight test. Sensor data will then be routed to the PHM system for fault diagnosis and RUL prediction. 


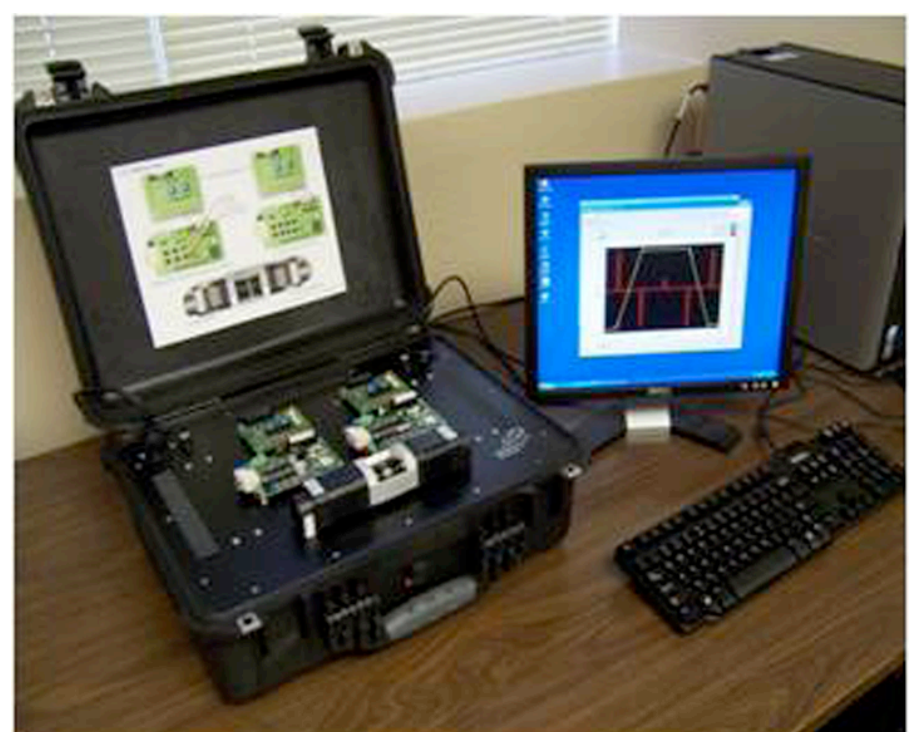

Figure 5. Ridgetop EMA emulator.

The prognosis of aging electronics and EMAs with seeded mechanical failures has gained the interest of the aviation industry, including actuator manufacturers who have expressed an interest in adding more phases to this research. A potential add-on to the research proposal is testing a hybrid aircraft control surface driven by both a conventional hydraulic actuator and an EMA. The RTIP Wiki will be used to generate an additional scenario linked to this test.

The RTIP Wiki was used to link the IVHM milestones aimed at validating methodologies and tools for the prognosis of failures associated with aircraft components and subsystems implicated by adverse events to assets available to the IVHM team to conduct the PET research. The wiki was also instrumental in identifying another test that could be performed on the same flight platform at the same time as the PET study. The FLEA test stand will be flown on the same flight as the PET's EMA emulator, which will further minimize the cost to the IVHM Project. This combined effort of two already collaborative research ventures demonstrates a benefit of using the IVHM RTIP Wiki to identify synergistic test opportunities.

\section{E. Structural Health Monitoring Flight Test}

A proposal to conduct a structural health monitoring flight test was also generated using the RTIP Wiki. The test seeks to use the NASA Langley Research Center (LaRC) Tributary Analysis Monitoring System (TAMS) on a flight-test bed in order to test tributary analysis across aircraft subsystems and gather vehicle-wide state data. The TAMS box will be integrated into a flight-test aircraft to detect structural anomalies using input from existing and state-of-the-art sensing technologies installed throughout the aircraft. The research meets the detection theme IVHM milestone to demonstrate the application of multiple complementary sensor technologies for the detection of damage or degradation to airframe structural components. ${ }^{3}$ For this particular test, the TAMS box will be installed on a NASA DFRC F-18 (McDonnell Douglas now The Boeing Company, Chicago, Illinois) research aircraft and will gather strain gauge data from the flight-test instrumentation. The TAMS box will be a passive ride-along payload on the F-18. During the flight, nominal operations will be conducted under several specified flight conditions. Data (flight parameters as well as data from the TAMS box) will be sent for download, and will be retrieved and analyzed post-flight. Analysis of data from a single vehicle is necessary in order to progress towards fleet-wide tributary analysis. The RTIP Wiki has been successfully used to link the IVHM milestone to assets (the LaRC TAMS box and DFRC F-18 aircraft) and personnel located across multiple NASA Centers in order to develop a test plan and perform the research.

\section{Summary and Conclusion}

The electronic, web-based IVHM RTIP Wiki platform allows multiple users across multiple NASA Centers, as well as industry, university, and other government agency partners to access the information needed to readily meet IVHM research objectives. The RTIP Wiki contains sections on the IVHM research themes, capabilities and assets for performing IVHM research, integration and test plans, and documentation. IVHM theme-cognizant engineers 
use the wiki to develop research scenarios that enable common resource research and testing to occur. The joint test opportunities combine assets and personnel from NASA, industry, academia, and other government agencies. This allows higher TRL research to occur without adding a significant cost increase to the research budget. The research scenarios developed using the RTIP Wiki increase communication, collaboration, and awareness within the IVHM Project and the synergistic research opportunities allow IVHM research to advance further than what would be achievable with isolated efforts. A research scenario is linked to the appropriate IVHM milestones and resources detailed in the RTIP Wiki, reviewed by the research team members, and integrated into a collaborative test strategy. In addition to a description of the method of creating these collaborative research scenarios and test opportunities, examples of the successful development and implementation of cooperative research using the IVHM RTIP Wiki have been described, including a commercial turbofan engine ground test, a fleet level data-mining effort, an EMA flight test, a structural health monitoring flight test, and an aging electronics/actuator health management flight test. Each research scenario has benefited by using the RTIP Wiki in identifying joint test opportunities, planning the research, and integrating the collaborative efforts.

\section{Acknowledgments}

The authors wish to thank the NASA IVHM and RTIP teams, including the IVHM Principal Investigator, Project Scientist, Project Manager, APIs, cognizant engineers, chief engineers and researchers.

\section{References}

${ }^{1}$ Roudakov, A. S., Semenov, V. L., and Hicks, J. W., "Recent Flight Test Results of the Joint CIAM-NASA Mach 6.5 Scramjet Flight Program," NASA TP-206548, April 1998.

${ }^{2}$ Rombough, D. H., "Creating a Joint Aviation Engineering and Flight Test Organization," Strategy Research Project, U.S. Army War College, Carlisle, PA, March 2007.

${ }^{3}$ Srivastava, A. N., Mah, R. W., and Meyer, C., "NASA Integrated Vehicle Health Management Technical Plan Ver. 2.03," NASA, Aeronautics Research Mission Directorate, Aviation Safety Program [online document], URL: http://www.aeronautics. nasa.gov/nra_pdf/ivhm_tech_plan_c1.pdf, 2 November 2009, [cited 2 March 2010].

${ }^{4}$ National Research Council Steering Committee for the Decadal Survey of Civil Aeronautics, Decadal Survey of Civil Aeronautics: Foundation for the Future, The National Academies Press, Washington, DC, 2006.

${ }^{5}$ National Science and Technology Council, Aeronautics Science and Technology Subcommittee, "National Plan for Aeronautics Research and Development and Related Infrastructure," December 2007.

${ }^{6}$ Joint Planning and Development Office, "Next Generation Air Transportation System Integrated Work Plan," Ver. 1.0, [online document], URL: http://www.jpdo.gov/iwp.asp [cited 2 March 2010]. 